\title{
Casco inteligente de seguridad industrial para la prevención de accidentes y enfermedades ocupacionales
}

\section{(Industrial Safety Smart Helmet for the Prevention of Accidents and Occupational Diseases)}

\author{
Henry Nelson Aguilera Vidal ${ }^{1}$, Franklin Landerson Gallegos Ramírez ${ }^{1}$, Anabell Martha Rea \\ Freire $^{1}$, Michael Nilo Galeas ${ }^{1}$ \\ ${ }^{1}$ Facultad de Ciencias de la Ingeniería, Universidad Técnica Estatal de Quevedo, Quevedo, Ecuador \\ haguilera@uteq.edu.ec,franklin.gallegos2015@uteq.edu.ec,anabel.rea2015@uteq.edu.ec, \\ michael.galeas2015@uteq.edu.ec
}

\begin{abstract}
Resumen: La seguridad industrial es un relevante para las empresas debido al uso contante de herramientas, maquinarias y equipos, creando un entorno de trabajo lleno de riesgos y peligros para los trabajadores. Por ello, hay normas que exigen la utilización de los EPI tales como el casco para prevenir accidentes y enfermedades ocupacionales. Sin embargo, en la actualidad no existe una solución tecnológica en este ámbito que proporcione en tiempo real información tanto al trabajador como al personal médico y al departamento de seguridad industrial, y que alerte al trabajador sin equipos adicionales o la necesidad de personal especializado en mediciones ambientales como túneles o minas. Por ello, en este trabajo se propone un casco de seguridad inteligente que podría ayudar a reducir el índice de accidentabilidad de los trabajadores. El casco utiliza componentes electrónicos como dispositivos de alerta y puede analizar niveles nocivos de gases, con el fin de prevenir enfermedades ocupacionales a corto y largo plazo a consecuencia de la actividad que realizan los trabajadores.
\end{abstract}

Palabras clave: casco inteligente, salud ocupacional, seguridad industrial.

\begin{abstract}
Industrial safety is relevant for companies due to the constant use of tools, machinery and equipment, creating a work environment full of risks and dangers for workers. For this reason, there are regulations that require the utilization of PPE such as the helmet that you use to accidents and occupational diseases. However, at present there is no technological solution in this area that provides information in real time to both the worker and the medical staff and the industrial safety department, and that alerts the worker without additional equipment or the need for specialized personnel in measurements environmental such as tunnels or mines. For this reason, this work proposes an intelligent safety helmet that could help reduce the accident rate of workers. The helmet uses electronic components as warning devices and analyzes harmful levels of gases, in order to prevent occupational diseases in the short and long term as a result of the activity carried out by workers.
\end{abstract}

Keywords: smart helmet, occupational health, industrial safety.

\section{INTRODUCCIÓN}

La seguridad industrial es un tema de gran importancia para las empresas, pues debido a la gran cantidad de herramientas, maquinarias y equipos que se manejan se crea al mismo tiempo un entorno de trabajo lleno de riesgos y peligros para los trabajadores. Es por esto que existen 
normas que obligan el uso de Equipos de Protección Personal (EPP) [1] en todo momento. En base a lo anterior, podemos decir que es importante innovar continuamente la efectividad y confiabilidad de estos equipos de seguridad, y de esta manera asegurar la integridad del trabajador [2].

En la industria minera por ejemplo [3], la construcción de túneles y de obras subterráneas implica riesgos para todas las partes implicadas así como para otras no directamente involucradas, debido a las incertidumbres inherentes a estos proyectos. Si no se toman medidas o acciones necesarias para disminuir los riesgos en futuros proyectos de minería túneles y galerías, el índice de accidentabilidad en los trabajadores se incrementaría durante su ejecución de las actividades. Para disminuir los riesgos que ocurren en trabajos subterráneos de minería, galería y túneles se necesitan cascos de seguridad industrial inteligentes que ayuden a prevenir accidentes y enfermedades ocupacionales en la industria minera y de construcción.

En la actualidad no existen soluciones tecnológicas en el campo de la seguridad industrial y salud en el trabajo, que brinde información en tiempo real tanto al trabajador como al personal médico y departamento de seguridad industrial, y que a la vez alerte al trabajador sin equipos adicionales o la necesidad de personal especializado en mediciones ambientales [4] como dióxido de carbono, gas metano, temperatura, humedad del lugar de trabajo en túneles y minería, similar a lo que se hace en ambientes cerrados [5].

Por ello, con este proyecto se busca la implementación de mejoras a estos equipos, orientadas a la interacción inteligente tanto con su entorno como con el usuario, añadiendo una serie de características pensadas para aumentar al máximo la integridad y seguridad de los operarios en el sector de la minería y la industria [2]. Así, el objetivo de este trabajo fue diseñar un casco de seguridad industrial "inteligente", que mediante el uso de sensores y actuadores tecnológicos, contribuya a la prevención de accidentes y enfermedades ocupacionales en la industria minera. La finalidad de diseñar este casco de seguridad inteligente es de reducir el índice de accidentabilidad de los trabajadores utilizando componentes electrónicos como dispositivos de alerta. También permitirá realizar un análisis de la calidad atmosférica específicamente de gases metano y dióxido de carbono, con el fin de prevenir enfermedades ocupacionales a corto y largo plazo a consecuencia de la actividad que realizan los trabajadores.

\section{METODOLOGÍA}

\subsection{Casco "inteligente"}

El casco diseñado cuenta con múltiples sensores entre ellos tres sensores de proximidad que se instalan en lugares específicos de un casco de seguridad. Estos sensores emiten una señal eléctrica en caso de encontrarse a menos de $50 \mathrm{~cm}$. De distancia de un objeto.

La señal es captada por un Arduino, el cual la envía a un motor vibratorio haciendo haciéndole actuar de manera inmediata. Así, a través de vibraciones localizadas, se le indica al usuario cuando un objeto se aproxima al trabajador. Además, la frecuencia de vibración se incrementa al reducir la distancia entre el objeto y el sensor. También, el casco permite realizar un análisis de la calidad atmosférica específicamente de gases metano y dióxido de carbono dando a conocer en tiempo real las concentraciones a la que está expuesto los trabajadores, cuenta con un sensor de DTH11 quien se encarga de tomar la lectura de temperatura, humedad a la que se encuentra laborando el trabajador, con el fin de prevenir enfermedades ocupacionales a corto y largo plazo a consecuencia de la actividad que realizan.

El sistema es alimentado por una batería externa de ion litio la cual está conectada al Arduino y al motor. Las conexiones entre el Arduino, sensores, motores y alimentación convergen en una placa PCB, ubicada junto al Arduino y la batería en una caja pequeña en la zona trasera del casco. 


\subsection{Métodos}

Éste proyecto se realizó mediante un estudio de tipo exploratorio, en el cual se analizó la factibilidad de la implementación de un prototipo de casco inteligente minero, con los diferentes métodos de ensayo y observaciones de campo a los trabajadores expuestos.

También, se recopilado información documental del modelo de casco a evaluar, así como como también en campo para la realización del estudio; es decir se tendrá que identificar a la población expuesta para evaluar mediante los métodos descritos en este trabajo.

\subsection{Materiales}

A continuación se listan los materiales empleados en el diseño del prototipo de casco inteligente.

- sensor ultrasónico

- motores vibrador

- resistencias

- Cables de conexión

- placa pre perforada

- porta baterías

- batería de ion litio 3000 M.A

- sensor de temperatura, humedad

- arduino omega

- buzzer

- Panel solar

- Leds t rojo

- Sensor de dióxido de carbono co2

- Sensor de gas metano CH4

- pantalla oled $12 \mathrm{c}$

- ESP8266 wifi comunicación

- Batería recargable 9v

- Interruptor

- Módulo de carga 5v

- El módulo controlador de motores

- Cautín

- Estaño

- Pasta de soldar

- Porta cables

- Pinza

- Pistola de silicona

- Cable de datos

\section{Equipos}

- Multímetro

- Laptop

- Impresora

- Memoria USB (8gb)

\section{Software}

- IBM spss statistics 25

- Arduino uno 
- Blynk

- Microsoft Word, Excel

\section{RESULTADOS}

Las figuras 1 y 2 muestras el resultado de la elaboración del casco de seguridad industrial inteligente incorporado dispositivos electrónicos como sensores de temperatura, humedad, dióxido de carbono, metano, ultrasónico, que permitan monitorear constantemente y reducir el incide de accidentabilidad.

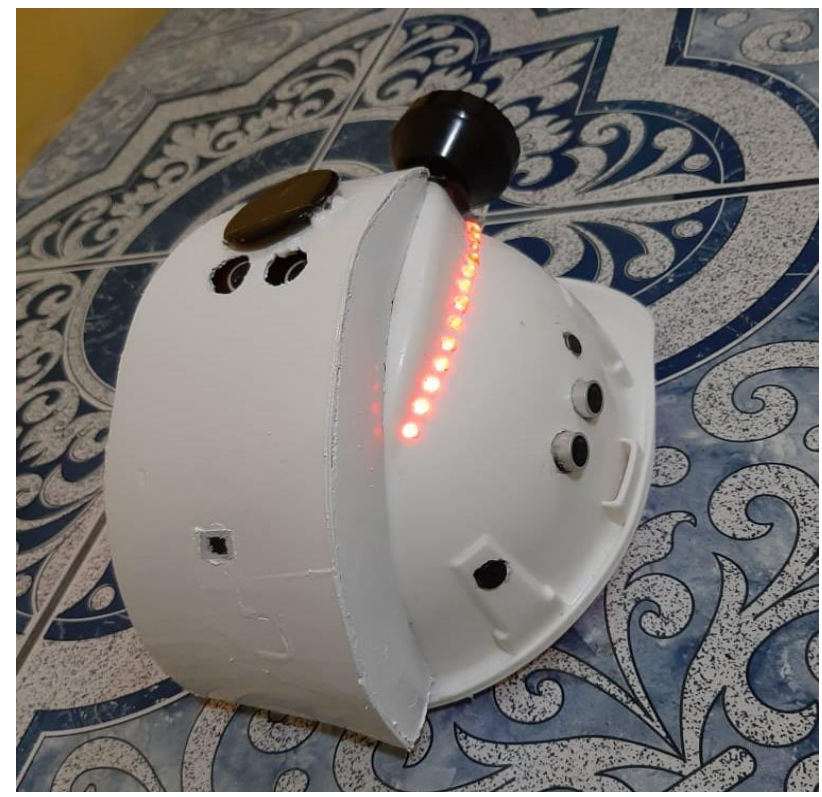

Figura 1. Vista lateral-posterior del casco propuesto.

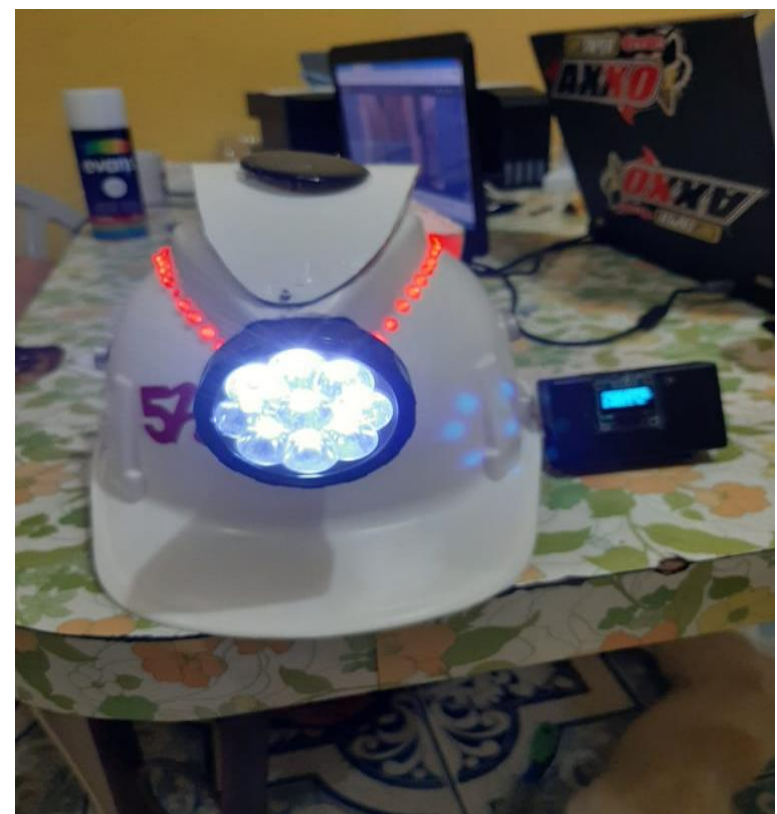

Figura 2. Vista frontal del casco propuesto.

Se realizaron pruebas de funcionamiento para determinar que todos los componentes electrónicos incorporados en el casco de seguridad industrial inteligente trabajan correctamente. 
Asimismo, se verificó que emitan los datos detectados por los sensores como se puede apreciar en la figura 3. En ella se observa la ejecución de la aplicación móvil que acompaña al casco. Esta aplicación fue desarrollada usando Blynk [6] y muestra en tiempo real los datos obtenidos de los sensores incorporados en el casco de seguridad industrial propuesto.

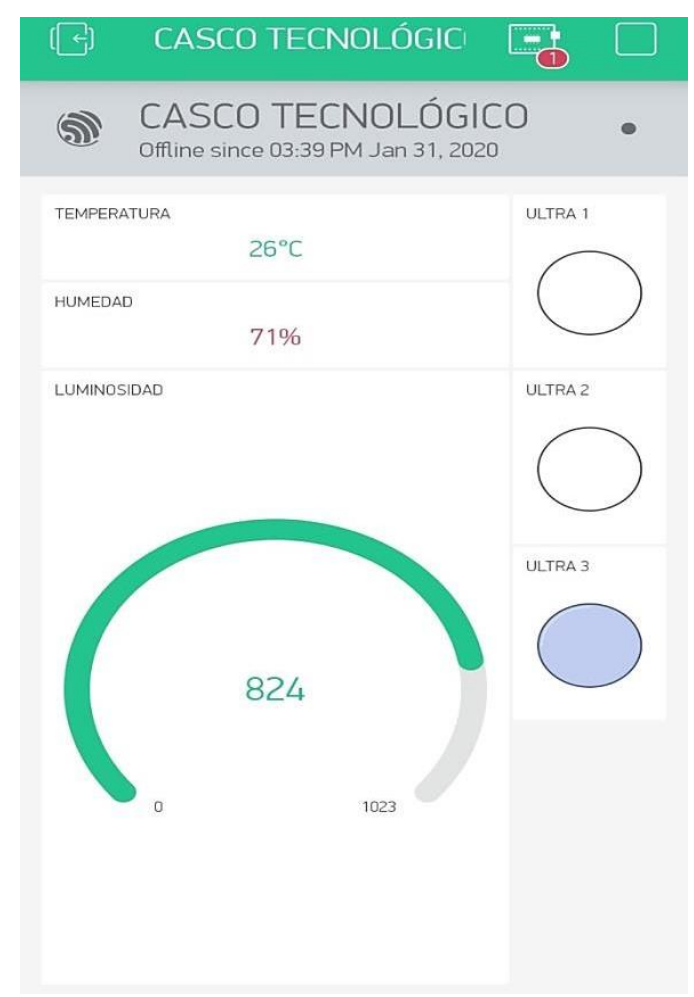

Figura 3. Aplicación móvil usando Blynk para visualización de valores leídos en el casco

Una vez verificado el funcionamiento de todos los componentes del sistema, se llevó a cabo una prueba piloto para ratificar este funcionamiento. Esta evaluación se llevó a cabo en una feria de seguridad industrial y salud ocupacional realizada en el campus de la institución a la que pertenecen los autores. En ella, se dispuso de un stand al que llegaron diferentes personas, fundamentalmente docentes y estudiantes relacionados al área de seguridad industrial. Al acercarse, se les proporcionó una explicación del casco para luego solicitarles colocárselo y probarlo. Finalmente, mediante una entrevista informal se requirió la opinión de ellos. En general, los comentarios fueron positivos, tales como: es una propuesta interesante, sería de utilidad para mineros, podría servir a otros tipos de trabajadores.

\section{CONCLUSIONES}

En este trabajo se ha descrito el diseño de un casco de seguridad industrial inteligente. Para ello, se seleccionaron los componentes electrónicos adecuados para la implantación, tales como sensores (de gases, ultrasónico, humedad, temperatura), Arduino, motor vibratorio, batería, panel solar, entre otros. Estos componentes permiten recopilar los datos obtenidos en el lugar de trabajo.

Se incorporó los sensores electrónicos al casco de seguridad industrial, obteniendo un funcionamiento correcto de los componentes. De esta manera se ha innovado un casco de forma tradicional a un casco inteligente. Gracias a la función de los sensores se obtienen datos específicos que permiten conocer los riesgos a los que están expuestos los trabajadores y por ende dar una media de control, con el fin de minimizar el índice de aceptabilidad y el riesgo de obtener a corto o largo plazo una enfermedad ocupacional. 
Finalmente, se desarrolló una aplicación móvil que permite al departamento de seguridad monitorear constantemente donde se registran los datos obtenidos en tiempo real. Asimismo, el trabajador portará una pulsera, basada en Internet de las cosas, de forma inalámbrica para visualizar la humedad y temperatura ambiente a la que está laborando, así como gases de dióxido carbono y gas metano, distancia de objetos para evitar accidentes mediante un sistema de alarma que se activa al detectar un objeto próximo a impactarse dando como aviso en forma de vibración en el casco y un sonido emitido por un "buzzer" que es percibido por el trabajador.

Teniendo en cuenta que solo se ha realizado una evaluación final, a futuro se espera llevar a cabo una nueva evaluación idealmente en un ambiente real para el cual el casco fue diseñado. Asimismo, se espera incorporar nuevas funcionalidades, como por ejemplo, proporcionar feedback táctil mediante la pulsera y detectar niveles nocivos de otros gases.

\section{REFERENCIAS}

[1] M. Abrego, S. Molinos y P. Ruíz, «Equipos de protección personal,» ACHS, vol. 32, 2000.

[2] Díaz.B, «Fablab.uchile,» 28 Septiembre 2018. [En línea]. Available:

http://www.fablab.uchile.cl/proyectos/322/casco-de-seguridad-proxihelmet/.

[3] J. H. Herbert, Seguridad, salud y prevencion de riesgos en mineria, Madrid, España, 2008.

[4] F. A. Cruz Salguero, Análisis de factibilidad de la implementación de un modelo de casco inteligente en trabajos de galerías y túneles, Quito, Ecuador: Universidad Internacional SEK, 2018.

[5] O. Erazo Moreta, Á. R. Santana Sornoza, M. J. Vera Alarcón y B. W. Oviedo Bayas, «Monitorización de gases contaminantes en ambientes cerrados usando WSN para la toma de acciones preventivas,» Universidad y Sociedad, vol. 12, nº 3, pp. 116-122, 2020.

[6] Blynk, «Blynk - IoT platform for businesses and developers,» [En línea]. Available: https://blynk.io/. [Último acceso: 2020]. 